\title{
Synthesis of Carbon Materials with Abnormally High Specific Surface Area
}

\author{
A.V. Melezhik, G.V. Smolsky, A.D. Zelenin, \\ E.A. Neskoromnaya*, O.V. Alekhina, A.E. Burakov, A.G. Tkachev \\ Tambov State Technical University, 106, Sovetskaya st., Tambov, 392000, Russia \\ * Corresponding author. Tel.: +7(953) 70783 28. E-mail: Lenok.n1992@mail.ru
}

\begin{abstract}
As a rule, carbon materials with developed energy are produced by physical or active activation of carbon-containing precursor materials. Physical activation is usually carried out at a temperature of $800-900{ }^{\circ} \mathrm{C}$. For chemical activation, many reagents have been proposed - phosphoric acid, zinc chloride, potassium sulfide, sodium hydroxide, potassium hydroxide, potassium carbonate, and others. All these techniques are aimed at increasing the specific surface and porosity of the original carbon nanomaterials.

The presented paper describes the synthesis technology for substances, presumably of a cumulene structure, based on the polycondensation of hexamethyltetramine in anhydrous sulfuric acid. A change in the heat treatment modes makes it possible to intensify the polymerization process, which results in synthesis products that are insoluble in an aqueous medium. Optionally, carbon nanotubes were added to the reaction system.

The substances obtained were activated with potassium hydroxide. The analysis of the specific surface area of the synthesized materials showed extremely high values $\left(4288-5346 \mathrm{~m}^{2} / \mathrm{g}\right)$. Increasing the ratio of potassium hydroxide to the initial carbon substance leads to increasing the pore width. The addition of carbon nanotubes to the initial substance dramatically increasesthe pore width. The activated materials can be used as effective adsorbents and electrode materials for chemical current sources.
\end{abstract}

\section{Keywords}

Effective sorbents; hexamethyltetramine; polycondensation; porosity; specific surface area; synthesis.

(C) A.V. Melezhik, G.V. Smolsky, A.D. Zelenin, E.A. Neskoromnaya, O.V. Alekhina, A.E. Burakov, A.G. Tkachev, 2019

\section{Introduction}

Carbon materials with a developed surface and porosity are widely used as adsorbents for water and air purification and protection against harmful chemical compounds, as well as electrode materials of chemical current sources, catalyst carriers, in medicine (hemosorbents, enterosorbents, dressing materials), water desalination systems, methane storage, and other areas [1]. For a number of applications, the best performance is achieved at the maximum possible values of the specific surface and porosity parameters. In this paper, the task is to synthesize such carbon materials.

\section{Materials and experimental methods}

The following starting materials were used in the present study:

- Urotropin $\mathrm{C}_{6} \mathrm{H}_{12} \mathrm{~N}_{4}$ (hexamethylenetetramine, HMTA), analytical grade;

- Sulfuric acid $\left(95 \% \mathrm{H}_{2} \mathrm{SO}_{4}\right)$, analytical grade;

- Oleum, technical grade, with the content of $24 \%$ free $\mathrm{SO}_{3}$. Diluted oleum containing $5 \% \mathrm{SO}_{3}$ was prepared by mixing calculated amounts of sulfuric acid and $24 \%$ oleum;

- Multiwalled carbon nanotubes Taunit-M produced by "NanoTechCenter" (Tambov). MWNTs 
were purified from rests of the catalyst by treatment with hydrochloric acid;

- Potassium hydroxide, analytical grade, was in the form of granules with a $\mathrm{KOH}$ content of $85 \%$ (the rest is water).

The synthesis of a carbon-containing substance of a supposed cumulene structure with the code name "polycumulen" was carried out by analogy with the synthesis of a soluble substance of a supposed cumulene structure described in $[2,3]$. However, in the present research, the heat treatment temperature was increased to $180-200{ }^{\circ} \mathrm{C}$ for deeper polymerization, which resulted in an insoluble product. The synthesis was carried out as follows. $45 \mathrm{ml}$ of $5 \%$ oleum was placed into a 2-liter beaker of heat-resistant glassunder the exhaust ventilation, and while cooling in a bath with cold water, at continuous stirring $30 \mathrm{~g}$ of HMTA was added in small portions. Then the beaker was placed in a thermostatic jacket, provided with thermal insulation and a thermocouple, and slowly heated on the electric cooker. When the temperature reached $110-120{ }^{\circ} \mathrm{C}$, an exothermic reaction took place with foaming of the reaction mixture due to the release of a certain amount of gas (analysis showed that it was mainly sulfur dioxide). The reaction mixture was kept for 2 hours at a temperature of $180-200{ }^{\circ} \mathrm{C}$. Foaming of the reaction mixture did not allow increasing the loading of initial reagents more than that. After cooling to room temperature, the product was poured with water, crushed and washed on the filter to a neutral $\mathrm{pH}$, and then dried at $110{ }^{\circ} \mathrm{C}$ to constant weight. Amount of produced substance was $17.91 \mathrm{~g}$ per one synthesis with the specified load. For the production of the material, synthesis was repeated several times. The energy dispersive analysis showed the presence of $11.1 \%$ of oxygen and $15.9 \%$ of sulfur in this substance, in addition to carbon ( $73.0 \%$ of the mass). Further, this substance will be conventionally denoted as C-110.

The synthesis of a composite, containing carbon nanotubes (CNTs), was carried out in a similar way. However, in the presence of CNTs, the foaming of the reaction mixture was less, which allowed the loading to be tripled (135 $\mathrm{ml}$ of $5 \%$ oleum, $90 \mathrm{~g}$ of HMTA). The initial mixing of HMTA with oleum was carried out with cooling, as described above. In this case, the reaction mixture partially crystallizes, probably due to the formation of the HMTA hydrosulfate at this stage. Then the beaker with the reaction mixture was heated $\left(60-70{ }^{\circ} \mathrm{C}\right)$ until the reaction mixture melted. A viscous clear liquid was formed. In this liquid, keeping the temperature in the specified range, $12 \mathrm{~g}$ of purified CNTs were added and mixed thoroughly until a homogeneous paste was formed. Then the beaker was placed into a thermostatic jacket and further processing was carried out, as described above for the synthesis without the addition of CNTs. After drying to constant weight at $110{ }^{\circ} \mathrm{C}, 64.17 \mathrm{~g}$ of product was obtained, in which the calculated content of CNTs was $18.7 \%$. Further, this substance will be referred to as C-CNT-110.

The substances C-110 and C-CNT-110 in the native form turned out to be inconvenient for activation, since their heat treatment with potassium hydroxide led to strong foaming, the reaction mixture got out of the reactor. To eliminate this effect, preliminary heat treatment of the samples was carried out for 4 hours at $500{ }^{\circ} \mathrm{C}$ in a tube furnace in a flow of argon. At the same time, volatile sulfur-containing compounds were released and the sulfur mass content in the products after pyrolysis was reduced to $0.3-0.5 \%$. The mass of products after heat treatment at $500{ }^{\circ} \mathrm{C}$ was (from the initial) $68.1 \%$ for material $\mathrm{C}$ and $74.2 \%$ for material C-CNT. The calculated mass content of CNTs after heat treatment of the material at $500{ }^{\circ} \mathrm{C}$ is $25.2 \%$.

For activation after heat treatment (at $500{ }^{\circ} \mathrm{C}$ ), the substance was ground to a size of particles less than $0.5 \mathrm{~mm}$. Then a portion of the substance $(5 \mathrm{~g})$ was placed in a steel beaker with an inner diameter of $68 \mathrm{~mm}$ and a height of $88 \mathrm{~mm}$ and potassium hydroxide pellets was added. The glass was purged with argon and closed with a cover containing an annular sluice through which, during the activation process, argon was passed to isolate the reaction zone from the atmosphere. This system was heated in a muffle furnace to $750{ }^{\circ} \mathrm{C}$ and held for 3 hours. After cooling to room temperature, the reaction mixture was poured with water, the precipitate was washed from alkali to neutral $\mathrm{pH}$, then kept for 24 hours with hydrochloric acid to dissolve the impurities of the metal compounds, washed again with water and dried at $110^{\circ} \mathrm{C}$ to constant weight. Further, the activated substances will be designated as C-500-A ( $x: 1)$, C-500-CNT-A ( $x: 1)$, where $x: 1$ is the mass ratio of pellets of $85 \%$ potassium hydroxide to the initial substance $(\mathrm{C}-500$ or C-CNT-500).

The surface and porosity parameters were measured by nitrogen adsorption at $77 \mathrm{~K}$ using an Autosorb-iQ instrument (Quantachrome Instruments). Mathematical models integrated into the instrument software were used to calculate the surface and porosity parameters. Since the studied materials have both micro and mesoporosity, the DFT model was used as the most suitable in this case. The variant of the DFT model was chosen based on the best fitting comparison with the experimental isotherm. For the samples studied, this was " $\mathrm{N}_{2}$ on carbon at $77 \mathrm{~K}$, slit/cylindrical pore, NLDFT equilibrium model". 


\section{Results and discussion}

As a rule, carbon materials with a developed surface are produced by physical or chemical activation of carbon-containing precursor materials. Physical activation is usually carried out with water vapor, carbon dioxide or their mixture at a temperature of about $800-900{ }^{\circ} \mathrm{C}$. For chemical activation, many reagents have been proposed - phosphoric acid, zinc chloride, potassium sulfide, sodium hydroxide, potassium hydroxide, potassium carbonate, and others. Materials with the highest specific surface area (up to $3000-4000 \mathrm{~m}^{2} / \mathrm{g}$ according to BET) were obtained by activation with potassium hydroxide at a temperature of $700-900{ }^{\circ} \mathrm{C}$. There are many works in this area, for example [4-12].

The BET specific surface area valuesof carbon materials exceeding the specific surface area of a graphene monolayer (about $2630 \mathrm{~m}^{2} / \mathrm{g}$ ) have beenstill considered as not physically realistic, to be rather effective values corresponding to the volume filling of micropores or ultramicropores. However, it was shown [13] that, from perforated graphene layers, the structures for which a geometric surface area reaches $5100 \mathrm{~m}^{2} / \mathrm{g}$ can be made. Microporous organometallic framework structures formed by metal ions and organic ligands, for which the effective BET specific surface area can exceed $4000 \mathrm{~m}^{2} / \mathrm{g}$ are also known [14]. Theoretically, the framework structures with a very high specific surface could be constructed from a pure carbon skeleton, however, such substances are not known to date.

At the same time, it can be assumed, that during the chemical activation of carbon materials, the structures made of perforated graphene layers can be obtained. This assumption is based on the fact, that the activation of carbon, in particular, graphene materials with potassium hydroxide, begins primarily at structural defects, and, therefore, the formation of perforated and possibly skeletal structures is quite likely. So, it is known, that defective graphene, obtained by thermal or microwave decomposition of graphene oxide, can be effectively activated with potassium hydroxide $[15,16]$. At the same time, graphite and graphene nanoplatelets with a highly ordered structure, obtained by exfoliating graphite, as shown by our experiments, do not lend themselves to high-temperature activation with $\mathrm{KOH}$, and the surface development and mass loss almost never occur. The same effect is known for carbon nanotubes - the more defective their structure, the better they can be activated with potassium hydroxide [17].

Earlier, we assumed that the carbon-containing substance formed during the polycondensation of
HMTA in anhydrous sulfuric acid or dilute oleum $[2,3]$ contains fragments of a polycumulene structure. The activation of such substances with potassium hydroxide has not been previously studied. It is possible that as a result of chemical activation, a framework structure, including polycumulene fragments, is formed with an anomalous high specific surface.

Figures $1, a-f$ show the pore size distribution for activated samples C-500-A $(2: 1,3: 1,4: 1)$ and C-CNT-500-A $(2: 1,3: 1,4: 1)$ synthesized as described above in the experimental part.

Table 1 shows the surface and porosity parameters of the samples, as well as a yield of the activated products in $\mathrm{wt} \%$ from the initial substances after $500^{\circ} \mathrm{C}$.

The table shows that the BET specific surface area of the samples C-500-A is very large and reaches an extremely high value of $5346 \mathrm{~m}^{2} / \mathrm{g}$ for the sample C-500-A (3:1). This specific surface area is much higher than the surface of the graphene layer $\left(2630 \mathrm{~m}^{2} / \mathrm{g}\right)$. It can be assumed that a framework carbon structure is formed based on perforated graphene layers or polycumulene fragments.

The figures displaythe increase of the pore width, which is naturally associated with the burning of the pore walls, when increasing the amount of activating reagent $(\mathrm{KOH})$ for all materials. Adding carbon nanotubes to the composition of the activated material dramatically changes the pore size distribution in the direction of wider mesopores. The mass content of CNTscannot be determinedin the activated material, because carbon nanotubes in the activation conditions can also be activated with the burning of the masspart.

Interestingly, our attempts to produce wide-porous carbon materials by introducing CNTs into the original system dextrin-phenol-formaldehyde resin [18], as well as into the system dextrin-phenol-formaldehyde resingraphene oxide, did not give significant results. It can be assumed that for effective control of the pore size, carbon nanotubes should be well distributed in the matrix of carbon-containing material. Then the CNTs create a rigid framework, which, when a portion of the activated material is burned out, produces pores of an increased size. If the nanotubes are present in the form of aggregates, then a significant part of the volume of the activated material is out of contact with the nanotubes and effect of CNTs on the pore size is negligible. Apparently, the solution of HMTA and its polycondensation products in anhydrous sulfuric acid well wets the surface of carbon nanotubes, which leads to their disaggregation. 


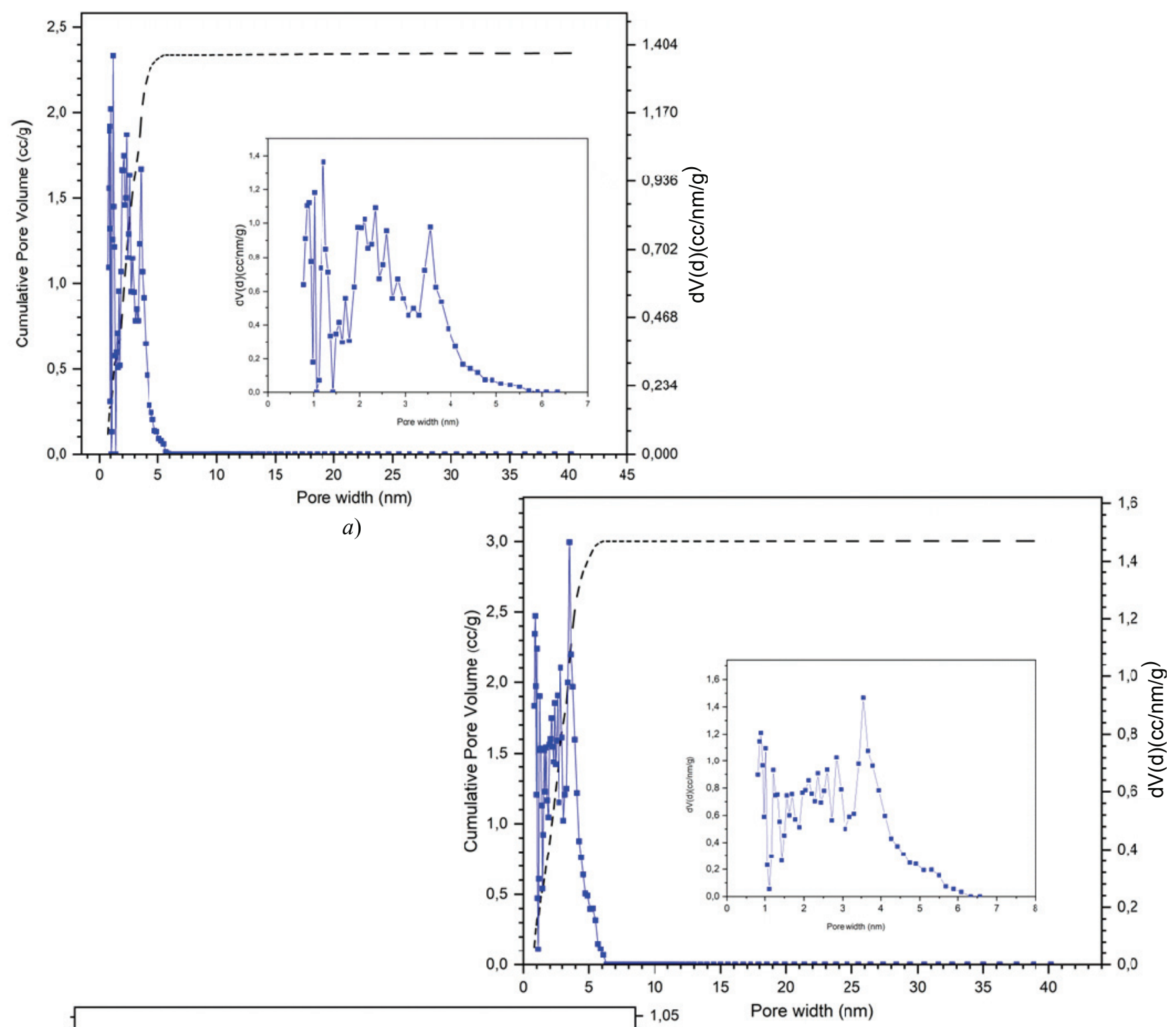

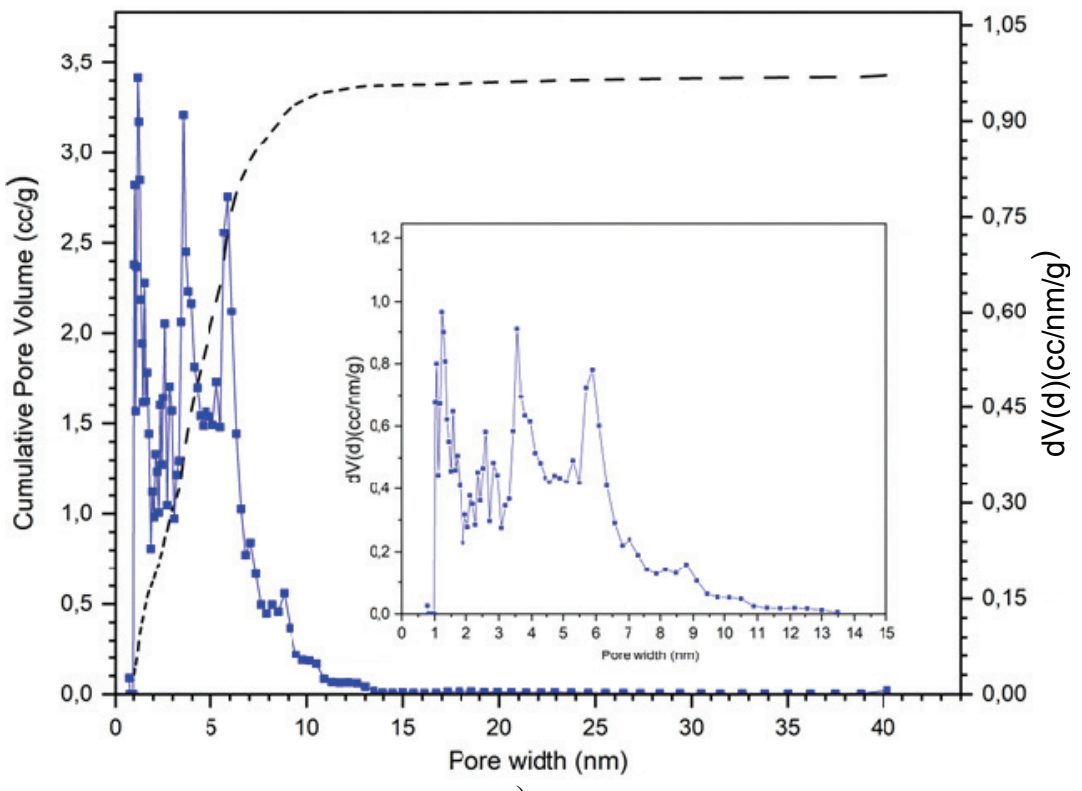

c)
Fig. 1. Integral and differential distribution of pore diameters for samples C-500-A $(a-c)$ and C-CNT-500-A $(\boldsymbol{d}-\boldsymbol{f})$ :

$a, d-(2: 1) ; b, e-(3: 1) ; c, f-(4: 1)$ 


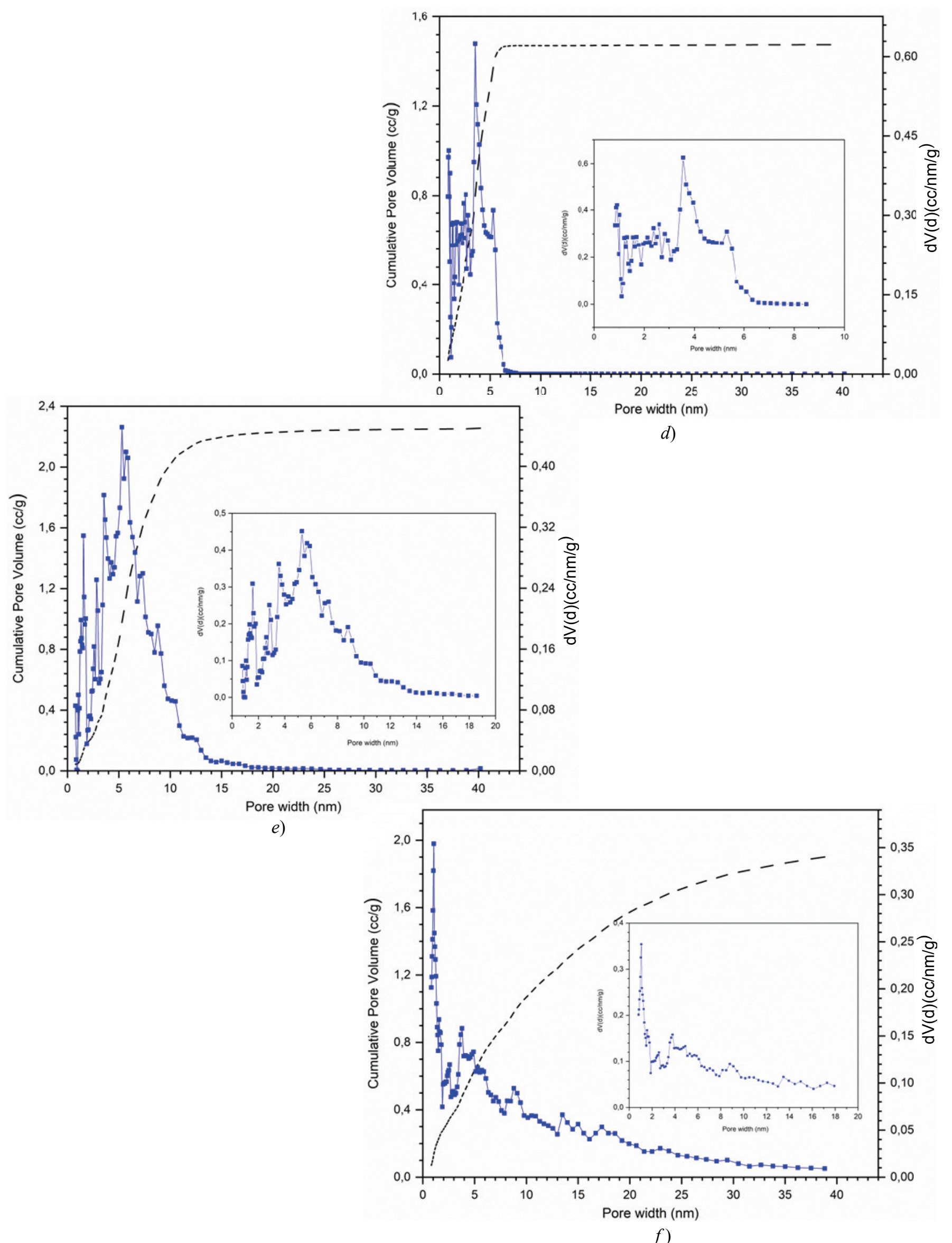


Surface and porosity parameters of samples of activated materials

\begin{tabular}{lcccccc}
\hline \multicolumn{1}{c}{ Sample } & $\begin{array}{c}\text { Yield, } \\
\mathrm{wt} \%\end{array}$ & $\begin{array}{c}S \text { BET, } \\
\mathrm{m}^{2} / \mathrm{g}\end{array}$ & $\begin{array}{c}S \text { DFT, } \\
\mathrm{m}^{2} / \mathrm{g}\end{array}$ & $\begin{array}{r}\text { Pore volume DFT, } \\
\mathrm{cm}^{3} / \mathrm{g}\end{array}$ & $\begin{array}{c}\text { Pore volume DFT, } \\
<2 \mathrm{~nm}, \mathrm{~cm}^{3} / \mathrm{g}\end{array}$ & $\begin{array}{c}\text { Total pore } \\
\text { volume, } \mathrm{cm}^{3} / \mathrm{g}\end{array}$ \\
\hline C-500-A(2:1) & 25.4 & 4491 & 3699 & 2.345 & 0.851 & 2.441 \\
C-500-A(3:1) & 21.8 & 5346 & 4272 & 3.004 & 0.886 & 3.121 \\
C-500-A(4:1) & 17.2 & 4288 & 3603 & 3.428 & 0.630 & 3.540 \\
C-500-УHT-A(2:1) & 38.8 & 2308 & 1894 & 1.477 & 0.336 & 1.531 \\
C-500-УHT-A(3:1) & 34.0 & 2024 & 1840 & 2.256 & 0.191 & 2.310 \\
C-500-УHT-A(4:1) & 29.3 & 1517 & 1433 & 1.980 & 0.285 & 2.087 \\
\hline
\end{tabular}

\section{Conclusion}

Thus, as a result of the research, the activated carbon materials with an anomalous high specific surface area were obtained, which makes it possible to assume the formation of a mesoporous carbon framework. The addition of carbon nanotubes to the initial system leads to a dramatic widening of the pores, which is probably caused by the formation of a framework of carbon nanotubes.

It should be noted that the use of HMTA and its polycondensation products effectively moisten the surface of the applied CNTs. This allows to significantly increase their disaggregation and as a result the uniform distribution in the porous structure of the composite. In turn, the uniformity of distribution has an important influence on the mechanical characteristics of the carbon skeleton, as well as the broadening of pores.

The materials obtained can be used as effective adsorbents and electrode materials for chemical current sources. In particular, materials containing carbon nanotubes can be used as adsorbents for large molecules, possibly biological molecules, which cannot be absorbed by conventional active carbons.

\section{Acknowledgements}

The research was funded by the Ministry of Education and Science of the Russian Federation (Project No.16.1384.2017/PCh).

\section{References}

1. Babkin A.V., Neskoromnaya E.A., Burakov A.E., Burakova I.V. A Kinetic on Copper (II) Adsorption from Aqueous Solutions on Graphene Oxide.Vestnik TGTU, vol.24, issue1, pp. 79-86.

2. Melezhik A.V., Alekhina O.V., Gerasimova A.V., Tkachev A.G. The Study of Polycondensation of Hexamethylenetetramine and the Properties of the Resulting Products. Vestnik TSTU, 2017, vol. 23, issue 3, pp. 461-470.

3. Patent RU 2661876. Tkachev A.G., Melezhik A.V., Alekhina O.V. Cumulene Substance, Method for its Production and Use. Publ. 07.20.2018.

4. Jiménez V., Sánchez P., Valverde J.L., Romero A. Influence of the Activating Agent and the Inert Gas (Type and Flow) Used in an
Activation Process for the Porosity Development of Carbon Nanofibers. Journal of Colloid and Interface Science, 2009, vol. 336, pp. 712-722.

5. Lozano-Castello D., Calo J.M., Cazorla-Amoros D., LinaresSolano A. Carbon Activation with $\mathrm{KOH}$ as Explored by Temperature Programmed Techniques, and the Effects of Hydrogen. Carbon, 2007, vol. 45, pp. 2529-2536.

6. Fierro V., Torne-Fernandez V., Celzard A. Highly Microporous Carbons Prepared by Activation of Kraft Lignin with KOH. Studies in Surface Science and Catalysis, 2007, vol. 160, pp. 607-614. doi: 10.1016/S0167-2991(07)80078-4.

7. Jun'ichi Hayashi, Mikihito Uchibayashi, Toshihide Horikawa, Katsuhiko Muroyama, Vincent G. Gomes. Synthesizing Activated Carbons from Resins by Chemical Activation with $\mathrm{K}_{2} \mathrm{CO}_{3}$. Carbon, 2002, vol. 40, pp. 2747-2752.

8. Chen X.S., Mcenaney B., Mays T.J., Alcaniz-Monge J., Cazorla-Amoros D., Linares-Solano A. Theoretical and Experimental Studies of Methane Adsorption on Microporous Carbons. Carbon, 1997, vol. 35, issue 9, pp. 1251-1258.

9. Liu B., Wang W., Wang N., Tong Au (P). C. Preparation of Activated Carbon with High Surface Area for High-Capacity Methane Storage. Journal of Energy Chemistry, 2014, vol. 23, issue 5, pp. 662-668.

10. Toshiro Otowa, Ritsuo Tanibata and Masao Itoh. Production and Adsorption Characteristics of MAXSORB: High-Surface-Area Active Carbon. Gas Separation \& Purification, 1993, vol. 7, issue 4, pp. 241-245.

11. Andrés A. García Blanco, Andrea F. Vallone, Sophia A. Korili, Antonio Gil, Karim Sapag. A Comparative Study of Several Microporous Materials to Store Methane by Adsorption. Microporous and Mesoporous Materials, 2016, vol. 224, pp. 323-331.

12. Aukett P.N., Quirke N., Riddiford S., Tennison S.R. Methane Adsorption on Microporous Carbons - a Comparison of Experiment, Theory, and Simulation. Carbon, 1992, vol. 30, issue 6, pp. 913-924.

13. Baburin I.A., Klechikov A., Mercier G., Talyzin A., Seifert G. Hydrogen Adsorption by Perforated Graphene. International Journal of Hydrogen Energy, 2015, vol. 40, issue 20, pp. 6594-6599.

14. Shen J., Sulkowski J., Beckner M., Dailly A. Effects of Textural and Surface Characteristics of Metal-organic Frameworks on the Methane Adsorption for Natural Gas Vehicular Application. Microporous and Mesoporous Materials, 2015, vol. 212, pp. 80-90.

15. Zhu Y., Murali Sh., Stoller M.D. [et al.] Carbon-based Supercapacitors Produced by Activation of Graphene. Science, 2011, vol. 332, issue 6037, pp. 1537-1541. doi: 10.1126/science. 1200770.

16. Murali Sh., Potts J.R., Stoller S. [et al.] Preparation of Activated Graphene and Effect of Activation Parameters on Electrochemical Capacitance. Carbon, 2012. vol. 50, issue 10, pp. 3482-3485.

17. Raymundo-Piñero E., Azaïs P., Cacciaguerra T. [et al.] $\mathrm{KOH}$ and $\mathrm{NaOHActivation} \mathrm{Mechanisms} \mathrm{of} \mathrm{Multiwalled} \mathrm{Carbon}$ Nanotubes with Different Structural Organization. Carbon. 2005, vol. 43, issue 4, pp. 786-795.

18. Patent RU 2620404. Tkachev A.G., Melezhik A.V., Solomakho G.V. The Method of Obtaining Mesoporous Carbon. Publ. 05.25.2017. 